\title{
Cerebral Artery Diameter in Inbred Mice Varies as a Function of Strain
}

\author{
Baogang Qian ${ }^{1,2 \dagger}$, Robert F. Rudy ${ }^{1,2 \dagger}$, Tianxi Cai ${ }^{3}$ and Rose Du ${ }^{1,2 *}$ \\ ${ }^{1}$ Department of Neurosurgery, Brigham and Women's Hospital, Boston, MA, United States, ${ }^{2}$ Harvard Medical School, \\ Harvard University, Boston, MA, United States, ${ }^{3}$ Biostatistics, Harvard T. H. Chan School of Public Health, Boston, MA, \\ United States
}

Many strains of mice are utilized in mouse models of cerebrovascular diseases. Variations in vascular anatomy between these strains has been documented and may influence the phenotype in stroke models. To address inter-strain variations in the circle of Willis anatomy, the diameters of internal carotid, posterior communicating, anterior cerebral, and middle cerebral arteries in 144 mice from 32 inbred strains were measured. Arterial diameters were analyzed as a function of animal weight, age, and strain. Variations in the structure of the circle of Willis across strains were observed and noted. While right-sided anterior cerebral arteries were significantly greater in diameter than their left-sided counterparts across most strains, variations in arterial diameter are strain specific. Adult mouse weight was not found to be associated with arterial diameter across strains, suggesting that cerebral artery size is associated with strain independently of weight. This study demonstrates strain dependent variations in the murine circle of Willis, which should be taken into consideration when studying mouse models of cerebrovascular diseases.

OPEN ACCESS

Edited by:

Pablo Blinder,

Tel Aviv University, Israel

Reviewed by:

Alan Urban

Neuroelectronics Research Flanders,

Belgium

Siu-Lung Chan,

University of Vermont, United States

*Correspondence:

Rose Du

rdu@bwh.harvard.edu

${ }^{\dagger}$ These authors have contributed equally to this work

Received: 20 October 2017 Accepted: 30 January 2018 Published: 20 February 2018

Citation:

Qian B, Rudy RF, Cai T and Du R (2018) Cerebral Artery Diameter in Inbred Mice Varies as a Function of

Strain. Front. Neuroanat. 12:10.

doi: 10.3389/fnana.2018.00010
Keywords: cerebral artery, cerebral circulation, mouse strain, variant anatomy, vascular anatomy

\section{INTRODUCTION}

Estimates suggest ischemic and hemorrhagic stroke each accounted for greater than three million deaths worldwide in 2013, making cerebrovascular disease the third leading cause of death in the world (Roth et al., 2015). Research into cerebrovascular disease relies heavily on relies heavily on animal models, particularly rodent models, as experimental systems (Casals et al., 2011). Mouse models have been used to study the effects of arterial occlusion (Hata et al., 1998; Orset et al., 2007), hemorrhagic stroke (Rynkowski et al., 2008; Bühler et al., 2014), intracranial aneurysms (Morimoto et al., 2002), and vascular etiologies of neurodegenerative disease (Nishio et al., 2010).

Differences in cerebrovascular anatomy among mouse strains have been previously reported at the level of the major cerebral vessels and also at the microvascular level (Ward et al., 1990; Barone et al., 1993; Maeda et al., 1998; Beckmann, 2000; Wellons et al., 2000; Zhang et al., 2010). Specifically, variations in the structure of the circle of Willis have been documented in different strains of mice (Ward et al., 1990; Barone et al., 1993; Beckmann, 2000). At the microvascular level, the number and diameters of collateral vessels between the anterior cerebral artery (ACA) and middle cerebral artery (MCA) have been shown to differ across strains (Maeda et al., 1998; Zhang et al., 2010). In addition, the susceptibility to ischemia and the percentage of total cortical surface area supplied by the MCA, ACA, and posterior cerebral artery (PCA) can differ significantly across mouse strains (Sheldon et al., 1998; Majid et al., 2000; Zhang et al., 2010; Du et al., 2015). Several strains have been utilized for murine stroke models (Hata et al., 1998; Orset et al., 2007; Zhang et al., 2010; Du et al., 2015). However, despite the evidence that cerebrovascular anatomy differs among 
mouse strains, reports on variations in the circle of Willis have been confined to a handful of strains. Given the widespread use of several mouse strains as model organisms in cerebrovascular disease and the potential for variations in cerebral artery diameter to influence infarct volume, there is a need for better understanding of the variations among strains.

Based on these previous findings, we hypothesized cerebral artery diameters would differ across mouse strains. Thus, the goal of this study was to assess and document variations in the circle of Willis in 32 inbred mouse strains. These 32 strains were chosen to provide a broad range of stroke susceptibility (Du et al., 2015).

\section{MATERIALS AND METHODS}

\section{Animal Care and Use Statement}

All animal care, housing, and experiments in this study were approved by and conducted in accordance with the Partners Healthcare System Policy on Humane Care and Use of Laboratory Animals from the Institutional Animal Care and Use Committee of the Harvard Medical Area Standing Committee on Animals. The experiments have been reported following the ARRIVE guidelines.

\section{Vessel Perfusion}

One hundred and forty-four male mice from 32 inbred strains, at least 8 weeks of age, were obtained from the Jackson Laboratory (Table 1). As there may be differences between male and female mice, we have restricted our study to male mice. Perfusion with India ink was performed to visualize cerebral blood vessels as previously described (Ward et al., 1990; Kitagawa et al., 1998; McGirt et al., 2002; Xue et al., 2014; Du et al., 2015). Briefly, mice were anesthetized with ketamine $[100 \mathrm{mg} / \mathrm{kg}$, intraperitoneal (IP)], xylazine (6 mg/kg, IP), and acepromazine (2 mg/kg, IP) (Patterson Veterinary Supply, St. Paul, MO) prior to transthoracic cannulation of the left ventricle and opening of the right atrium. Warm phosphate buffered saline was then perfused for 1-2 min, followed by $10 \%$ formalin (Fisher Scientific, Pittsburgh, PA) for $3 \mathrm{~min}$, and then a $5 \%$ gelatin (USB Corporation, Cleveland, $\mathrm{OH}$ )/saline solution with India ink (American Mastertech Scientific, Lodi, CA) at $55^{\circ} \mathrm{C}$ for $15 \mathrm{~s}$ (McGirt et al., 2002; Du et al., 2015). The phosphate buffered saline was kept warm to avoid large temperature fluctuations when the India ink perfusion is performed. The India ink solution is warmed to $55^{\circ} \mathrm{C}$ to keep the gelatin dissolved. All perfusates were delivered at pressures of $60-80 \mathrm{~mm} \mathrm{Hg}$ in order to maintain consistency. By using India ink perfusion, the diameters reported in this study represent the inner diameter of the vessel rather than the outer diameter. While it is possible for the vessel wall thickness to vary across strains, it is the inner diameter that is the more relevant parameter with regards to cerebral perfusion. Weight and age at the time of sacrifice prior to perfusion were recorded and mouse brains were removed for imaging.

\section{Vessel Measurement}

Photographs of the cerebral blood vessels perfused with India ink were obtained (Figure 1A). Standard human anatomical nomenclature was used to define murine artery names.
An illustration of the vascular nomenclature used is shown in Figure 1B. Vessels were measured using ImageJ v1.47 (Schneider et al., 2012). Measurements, made by an investigator blinded to the age and weight of the mouse, were obtained from the right and left internal carotid artery (ICA), M1 segment of the MCA, A1 segment of the ACA, and posterior communicating artery (PCoA). To standardize the location at which vessel were measured, measurements were taken between 100 and $300 \mu \mathrm{m}$ proximal (ICA diameter) and distal (M1 and A1 diameters) to the ICA bifurcation. PCoA measurements were taken proximal to the anastomosis with the first segment (P1) of the PCA, but due to overlapping tissues that may prevent good visualization, the measurements were taken $400-1200 \mu \mathrm{m}$ from the PCoA take off. The presence of P1 on the image was noted. In many cases, although P1 segments were not observed, ruling them out entirely was not possible given overlapping tissue, thus the terms observed and not observed were used.

\section{Principal Component Analysis}

Principal component analysis was then performed using data from the Mouse HapMap project (http://archive. broadinstitute.org/mouse/hapmap/). 132,285 Single nucleotide polymorphisms (SNPs) were extracted and those with a minor allele frequency $\leq 0.05$ were excluded, as were SNPs missing in $\geq 10 \%$ of strains leaving a total of 105,258 included SNPs. Only mice with complete datasets were used and the mean diameter of all vessels in each mouse was used as the phenotype. Using Plink v1.9 (Purcell et al., 2007) (http:// pngu.mgh.harvard.edu/purcell/plink/), principal component analysis was performed and the first two eigenvectors were plotted with ggplot2 in R (Wickham, 2009). Clusters were then labeled with vessel diameters categorized into four groups $(\geq 80-<100 \mu \mathrm{m}, \quad \geq 100-<120 \mu \mathrm{m}, \quad \geq 120-<140 \mu \mathrm{m}$, $\geq 140 \mu \mathrm{m})$.

\section{Heatmap}

Given C57BL/6J mice are commonly used in stroke models and were utilized by Hata et al. (1998) to study the association between weight and the diameter of filament need to occlude the MCA, we normalized the mean arterial diameters by dividing the mean vessel diameter for each strain by the average diameter in the C57BL/6J mice. The strains were subsequently clustered using a hierarchical clustering method based on the Euclidean distance matrix and complete agglomeration (gplots package in R Warnes et al., 2016).

\section{Statistical Analysis}

Statistical analysis was conducted in R v3.4 (R Core Team, 2017). Only complete (non-missing) data was used for the analysis. Wilcoxon signed-rank tests and Spearman correlations were used to determine differences in the size of and correlations between ipsilateral and the corresponding contralateral arteries. ANOVA between multivariate linear regression models with vessel size as the dependent variable and weight, age, and strain as covariates were used to evaluate the effect of each covariate on vessel diameter. $P$-values were adjusted 


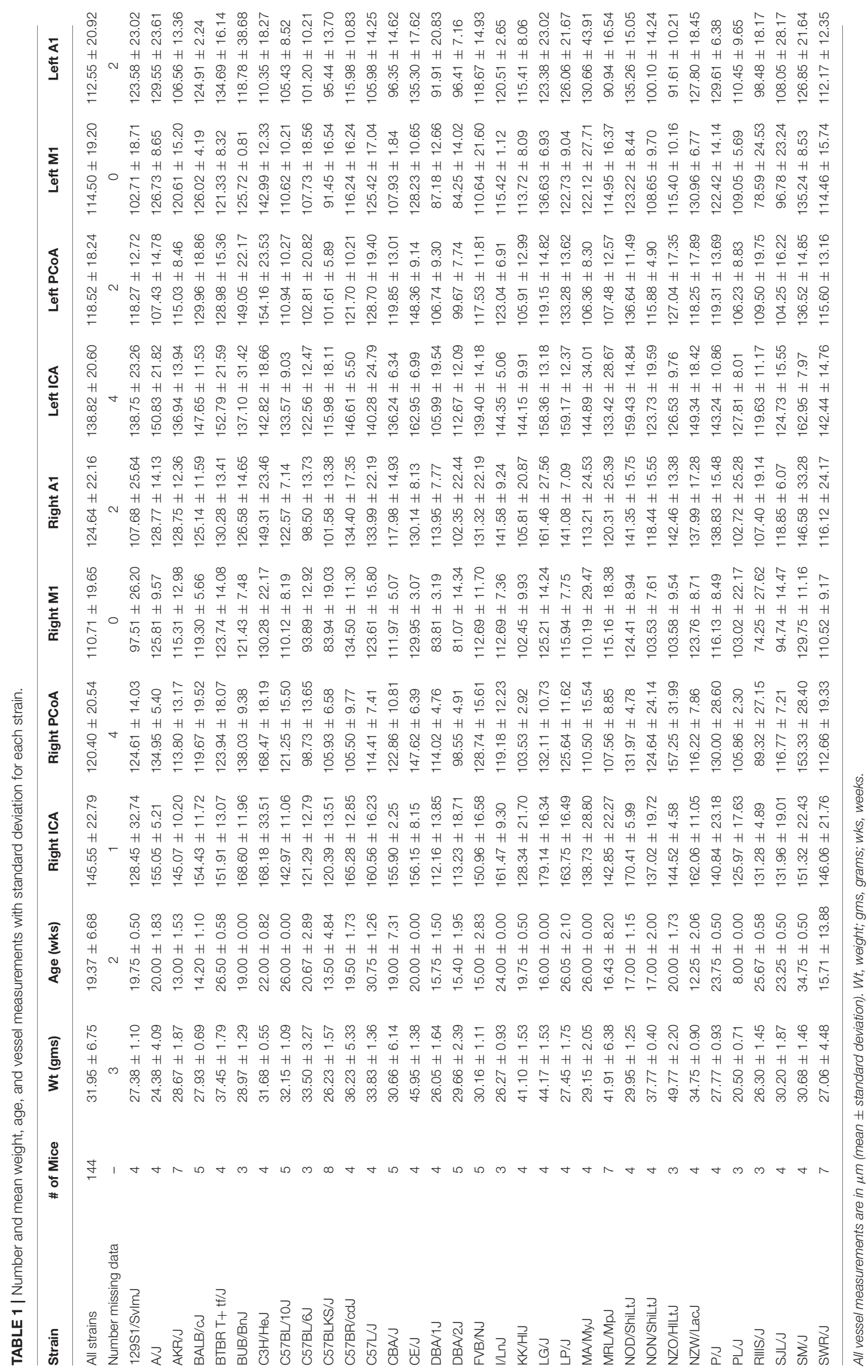




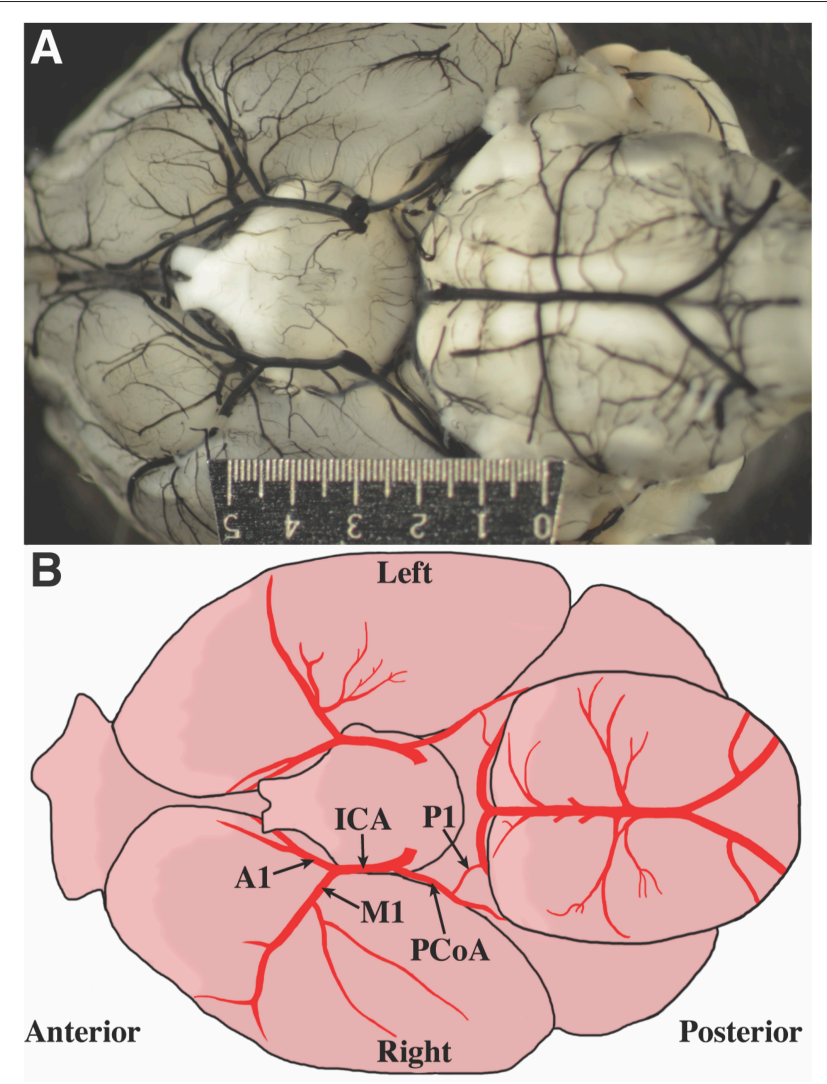

FIGURE 1 | Defining mouse anatomic nomenclature based on human anatomy. (A) Representative photograph of inferior surface of the murine brain. The ruler measures $5 \mathrm{~mm}$. (B) Illustration of the inferior surface of a murine brain. Blood vessel labels are shown for reference.

for multiple testing via the Benjamini-Yekutieli correction, which also accounts for correlation among the test statistics (Benjamini and Yekutieli, 2001). Adjusted $P$-values of less than 0.05 were considered significant. Additionally, the plyr (Wickham, 2011), dplyr (Wickham et al., 2017), tidyr (Wickham, 2017b), stringr (Wickham, 2017a), broom (Robinson, 2017), and RColorBrewer (Neuwirth, 2014) packages in $\mathrm{R}$ were utilized for data organization and figure generation.

\section{RESULTS}

\section{Vessel Size}

One hundred and forty-four male mice derived from 32 inbred strains were included in this study. Average measurements for all mice and for each strain separately are provided in Table $\mathbf{1}$ and distributions per vessel are shown in Figure 2A.

Differences in ipsilateral vessel diameters and left-sided vs. right-sided vessel diameters were examined (Table 2). Ipsilateral vessel comparisons were performed to determine relative sizes of vessels on each side, as a proxy for ascertaining the relative proportion of blood directed to the ACA, MCA, and posterior circulation from each ICA. ICAs were the largest vessel bilaterally. Right-sided A1 segments were larger than right-sided M1s, whereas on the left side these segments did not differ in size. Left PCoAs were larger than both left M1s and A1s, whereas right PCoAs were larger than right M1s but did not differ in size from right A1s. Right-sided A1s and ICAs were larger than their left-sided counterparts, whereas left-sided M1s were larger than those on the right. All four vessels measured demonstrated positive correlations between left and right, shown in Figure 2B, and Spearman correlation coefficient was statistically significant for all comparisons, with the exception of the right and left A1 segments (Supplementary Table 1).

We present in Figure $\mathbf{2 C}$ the heatmap of the mean arterial diameters for each strain normalized to those of C57BL/6J diameters. ANOVA comparing the multivariate models including weight, age, and strain and the models including only weight and age demonstrated that strain significantly contributes to the variation of vessel diameter at all arterial locations except for the left ICA and left A1 (Table 3). On the other hand, weight and age in adult mice were not significantly associated with vessel diameter at any location after accounting for strain (Table 3). Principal component analysis of the SNPs did not reveal clustering with respect to vessel diameters (Supplementary Figure 1).

\section{Anatomical Variants}

Numerous anatomic variants were observed during this study. The most common anomaly observed was absent or unilateral P1s (Figure 3A, Supplementary Table 2). Duplicated PCoAs were also observed, with two PCoAs originating from one ICA in four mice [A/J, FVB/NJ, and SM/J (2 mice) strains] (Figure 3B). MCA variation was the next most common site of variation. Accessory MCAs, namely, arteries originating in the distal ICA and traversing the typical MCA territory were observed in several mice across multiple strains (C57BL/6J, C57L/J, $\mathrm{CE} / \mathrm{J}, \mathrm{C} 57 \mathrm{BR} / \mathrm{cd}$, DBA/1J, FVB/NJ, MA/MyJ, NOD/ShiLt), NON/ShiLtJ, NZO/HlLtJ, PL/J, RIIIS/J, SWR/J) (Figure 3C). These additional MCA territory vessels varied in size from small to nearly identical to the ipsilateral MCA. Two mice from different strains (C57BR/cdJ, FVB/NJ) with fenestrated M1s were also observed (Figure 3D). Proximally narrow MCAs were also observed to varying degrees, with apparent distal dilatation (Figure 3E).

\section{DISCUSSION}

While mouse models are ubiquitous in cerebrovascular research, there has not been a study investigating variation in the size of murine cerebral arteries across a large number of inbred strains. In this study, we analyzed variations in the cerebral artery anatomy of 32 inbred mouse strains and found differences in vessel diameter based on both sidedness and strain.

\section{Nomenclature for Mouse Circle of Willis}

A consistent anatomic definition of murine circle of Willis anatomy is important for clarity and interpretation. However, 

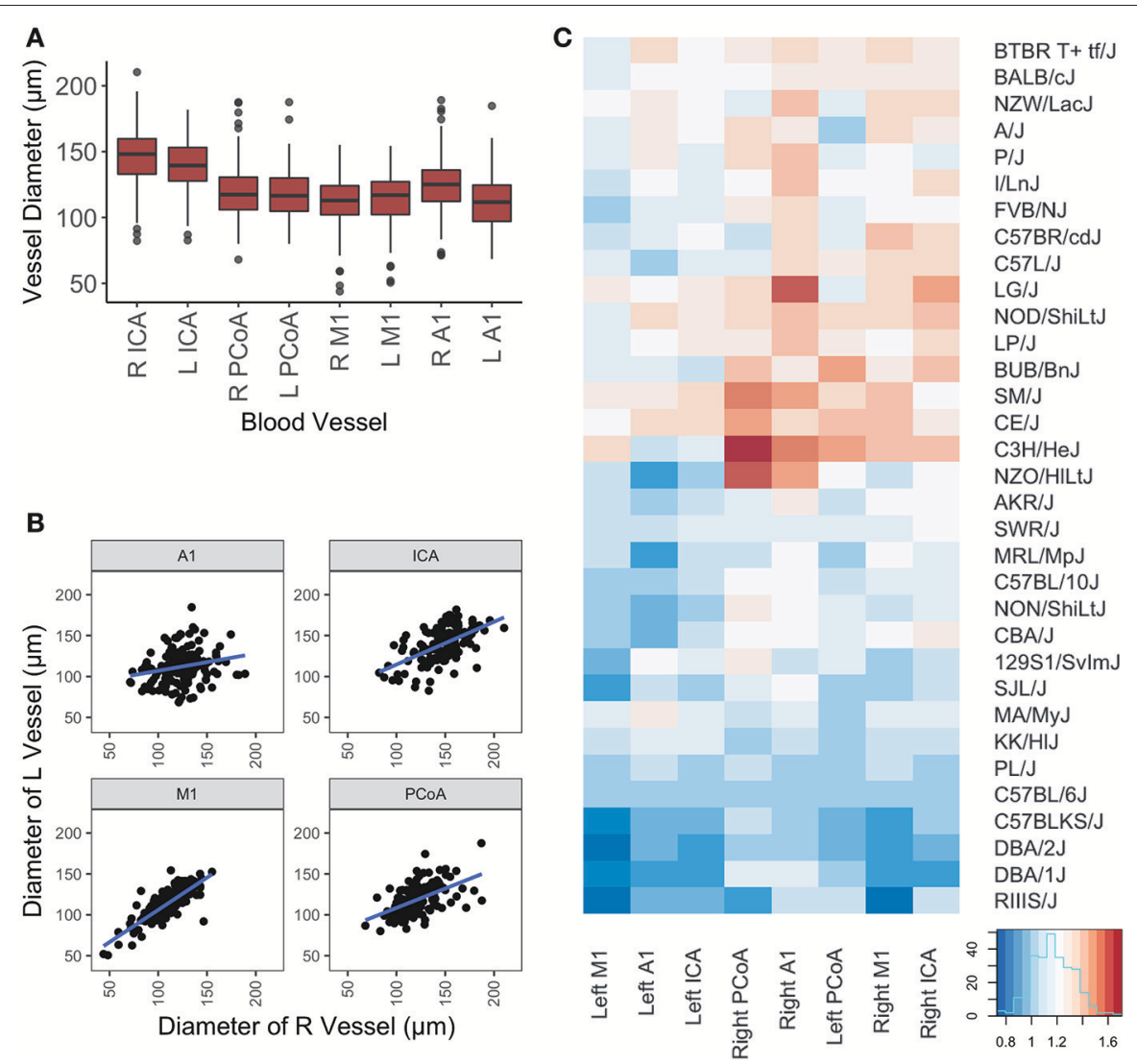

FIGURE 2 | Graphical representation of vessel diameters. (A) Boxplot depicting distribution of measurements for each vessel. Box represents 1st quartile through 3rd quartile, with line indicating median. Whiskers extend no more than $1.5 x$ the interquartile range. Data points beyond that are represented by a dot. (B) Scatterplot of left vs. right diameters for each vessel included in this study. Linear regression lines are depicted for each artery. (C) Graphical display of mouse vessel diameters by strain. Normalized mean vessel diameters were used to cluster strains, which are shown in a heatmap to provide visualization of the vascular patterns for each strain.

cerebral artery naming has not been consistent in the mouse literature. In particular, when compared to human anatomical labeling (Zeal and Rhoton, 1978; Schomer et al., 1994), the definition of the PCoA and P1 are often reversed (Barone et al., 1993; Kitagawa et al., 1998; Beckmann, 2000). In humans, the PCoA is defined as the vessel connecting the ICA to the PCA whereas the PCA arises from the basilar artery. P1 is the PCA segment starting with the origin of PCA until the anastomosis with PCoA. To maintain consistency, we used a naming system based on human anatomic nomenclature in this study.

\section{Variations of Cerebral Arteries in Different Locations}

Unsurprisingly, the ICA was found to be larger than other intracranial vessels. The right A1 segment was larger than the left A1 suggesting right A1 dominance. The ACA supplies the olfactory bulb, a robust structure in mice. In rats, asymmetry of the olfactory bulb has been reported, with the right side larger than the left (Heine and Galaburda, 1986). However, a study using MRI to investigate size asymmetry in mice did not find a statistically significant difference in the size of the right and left olfactory bulbs (Barbeito-Andrés et al., 2016). In addition to A1 asymmetry, we found the right ICA to be larger than the left ICA, and the left M1 to be larger than the M1. The MCA asymmetry may reflect the larger $\mathrm{A} 1$ on the left demanding more flow resulting in a smaller left $\mathrm{M} 1$, which would also explain the discrepancy in ipsilateral comparisons between the A1 and M1 on the right and left side. These data pose questions regarding the functional significance of arterial asymmetry, particularly that of A1.

\section{Variations of Cerebral Arteries across Different Inbred Strains}

Variations in diameters across strains in this study were not secondary to variations in weight, suggesting cerebral artery diameters are largely under genetic control in mice. The lack of clustering with mean vessel diameter in the principal component analysis should not be interpreted as evidence against predominant genetic control, but rather that vessel diameter phenotype is a complex phenotype.

These results appear to conflict with prior studies that found a significant, positive correlation between mouse weight and 
TABLE 2 | Comparisons between cerebral arteries with Benjamini-Yekutieli corrected $P$-values.

\begin{tabular}{|c|c|c|c|c|}
\hline Vessel 1 & Vessel 2 & $\begin{array}{c}\text { Wilcoxon signed rank } \\
\text { test estimate }[95 \% \\
\text { confidence interval] }\end{array}$ & $P$-value & $\begin{array}{c}\text { Adjusted } \\
P \text {-value }\end{array}$ \\
\hline R ICA & $\mathrm{R} M 1$ & 34.34 [31.91 to 36.87$]$ & $<0.001$ & $<0.001$ \\
\hline RICA & $\mathrm{R} A 1$ & 21.10 [18.78 to 23.37] & $<0.001$ & $<0.001$ \\
\hline R ICA & R PCoA & 26.62 [22.82 to 30.22$]$ & $<0.001$ & $<0.001$ \\
\hline $\mathrm{R} M 1$ & R A1 & $-13.34[-16.54$ to -10.36$]$ & $<0.001$ & $<0.001$ \\
\hline $\mathrm{R} M 1$ & R PCoA & $-9.70[-12.98$ to -6.44$]$ & $<0.001$ & $<0.001$ \\
\hline $\mathrm{R} A 1$ & R PCoA & $3.57[-0.11$ to 7.25$]$ & 0.055 & 1 \\
\hline LICA & L M1 & 24.32 [21.96 to 26.70$]$ & $<0.001$ & $<0.001$ \\
\hline L ICA & LA1 & 25.54 [23.30 to 27.80$]$ & $<0.001$ & $<0.001$ \\
\hline L ICA & LPCoA & 20.73 [17.37 to 23.97] & $<0.001$ & $<0.001$ \\
\hline L M1 & $\mathrm{LA} 1$ & $1.06[-1.83$ to 4.07$]$ & 0.466 & 1 \\
\hline L M1 & LPCoA & $-4.26[-7.13$ to -1.25$]$ & 0.007 & 0.135 \\
\hline $\mathrm{LA} 1$ & LPCoA & $-6.40[-9.43$ to -3.25$]$ & $<0.001$ & 0.003 \\
\hline R ICA & L ICA & 7.58 [4.01 to 11.02$]$ & $<0.001$ & $<0.001$ \\
\hline R PCoA & LPCoA & $1.30[-1.51$ to 4.21$]$ & 0.384 & 1 \\
\hline R M1 & L M1 & $-3.50[-5.46$ to -1.84$]$ & $<0.001$ & 0.003 \\
\hline R A1 & LA1 & $11.53[6.86$ to 16.04$]$ & $<0.001$ & $<0.001$ \\
\hline
\end{tabular}

$P$-values $<0.05$ are bolded

the filament diameter needed to produce an ischemic infarct (Hata et al., 1998). However, our study was limited to adult mice at least 8 weeks of age resulting in a small intrastrain variation in weight, whereas the study by Hata et al. utilized C57BL/6J mice $(N=35)$ with weights ranging from 18 to $33 \mathrm{~g}$ suggesting that some of these mice were younger in age than the ones included in this study. Large variations in weight, indicative of growing mice, likely do influence blood vessel diameters within a strain. However, strain, but not weight, was found to account for vessel variations in a multivariate model in adult mice. Thus, the dependence of vessel size on strain occurs independently of the mean weight of adult mice in that strain.

Zhang et al. reported differences in the percentage of brain surface perfused by large cerebral arteries across inbred mouse strains (Zhang et al., 2010). However, we found that some strains with relatively small areas supplied by the MCA according to Zhang et al. (e.g., NOD/ShiLtJ and NZW/LacJ) have large M1 diameters, suggesting that the size of the arterial territory may not be driven by the diameter of the vessel. While the diameter of arteries does not necessarily correspond to the percent of the brain surface area perfused, the study by Zhang et al. provides further evidence that variations in cerebrovascular anatomy are driven by strain.

Variation in diameters of major cerebral arteries amongst mouse strains has implications for occlusive ischemic models, including MCA occlusion via filament. The recent IMPROVE guidelines on appropriate utilization of in vivo ischemia modeling recommend using the smallest diameter filament that produces a reliable infarct (Percie du Sert et al., 2017). These data suggest that strain should be considered when choosing
TABLE 3 | ANOVA of multivariate models including the explanatory variables strain, weight, and age compared to a multivariate model with one of those variables removed.

\begin{tabular}{|c|c|c|c|}
\hline Artery & Variable of interest & Raw $P$-value & Adjusted $P$-value \\
\hline Right ICA & Strain & $<0.001$ & $<0.001$ \\
\hline Right PCoA & Strain & $<0.001$ & $<0.001$ \\
\hline Right M1 & Strain & $<0.001$ & $<0.001$ \\
\hline Right A1 & Strain & $<0.001$ & 0.005 \\
\hline Left ICA & Strain & $<0.001$ & 0.003 \\
\hline Left PCoA & Strain & $<0.001$ & $<0.001$ \\
\hline Left M1 & Strain & $<0.001$ & $<0.001$ \\
\hline Left A1 & Strain & $<0.001$ & 0.011 \\
\hline Right ICA & Weight & 0.402 & 1 \\
\hline Right PCoA & Weight & 0.763 & 1 \\
\hline Right M1 & Weight & 0.802 & 1 \\
\hline Right A1 & Weight & 0.787 & 1 \\
\hline Left ICA & Weight & 0.992 & 1 \\
\hline Left PCoA & Weight & 0.060 & 1 \\
\hline Left M1 & Weight & 0.720 & 1 \\
\hline Left A1 & Weight & 0.367 & 1 \\
\hline Right ICA & Age & 0.258 & 1 \\
\hline Right PCoA & Age & 0.087 & 1 \\
\hline Right M1 & Age & 0.642 & 1 \\
\hline Right A1 & Age & 0.030 & 0.853 \\
\hline Left ICA & Age & 0.141 & 1 \\
\hline Left PCoA & Age & 0.614 & 1 \\
\hline Left M1 & Age & 0.637 & 1 \\
\hline Left A1 & Age & 0.461 & 1 \\
\hline
\end{tabular}

Each row indicates a separate ANOVA for the respective vessel location. P-values were adjusted using the Benjamini-Yekutieli procedure. P-values $<0.05$ are bolded.

initial filament diameters when performing the MCA occlusion model.

\section{Variations in Circle of Willis Anatomy}

We observed several anatomic variants during the course of the study. Unilateral or bilateral absence of P1 was common. In our study, no BTBR T+tf/J or RIIIS/J mice were found to have visible bilateral P1 segments, whereas A/J, AKR/J, C57BL/10J, CE/J, $\mathrm{DBA} / 2 \mathrm{~J}$, and $\mathrm{P} / \mathrm{J}$ mice all had observable bilateral P1 segments. Variations in P1 presence are significant for stroke research as PCA territory infarctions could occur with anterior circulation occlusion when there is an absence of P1. This may contribute to the variation in infarct size in an MCA occlusion model. Furthermore, the absence of $\mathrm{P} 1$ segments results in a non-intact circle of Willis which is known to increase the risk of hypotensive infarcts in humans (Schomer et al., 1994). MCA variation has been documented previously in over $17 \%$ of Sprague-Dawley rats (Fox et al., 1993). Duplicated and accessory MCAs have also been reported in humans, some of which arose from the proximal ACA and others from the distal ICA (Komiyama et al., 1997, 1998; Chang and Kim, 2011; Liu et al., 2015). Case reports of human patients with accessory MCAs and MCA occlusion report favorable outcomes, suggesting these vessels can perfuse at least some of the at-risk territory following MCA occlusion 

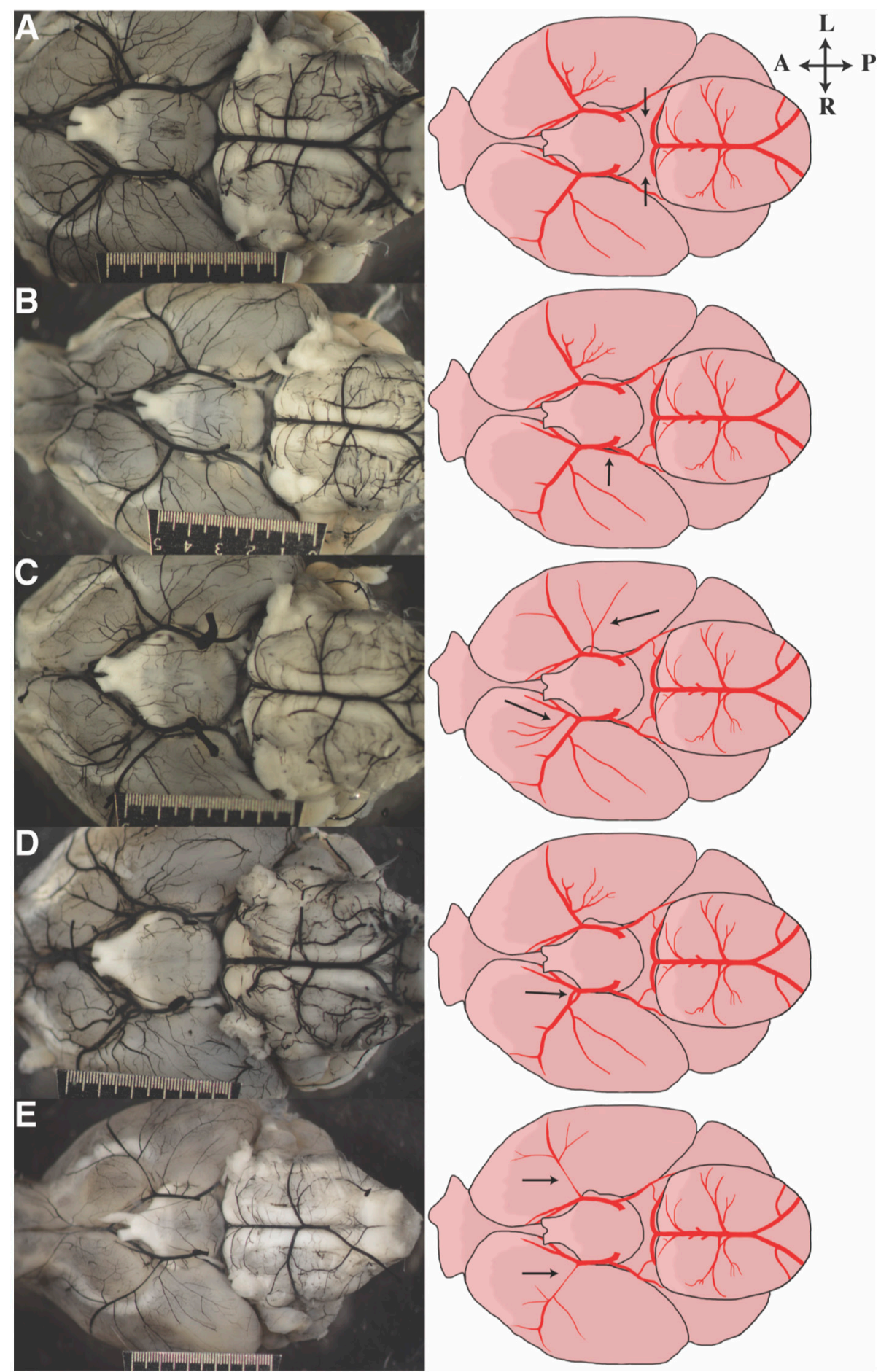

FIGURE 3 | Representative images of anatomic variants observed in our cohort of mice. Photograph on the left corresponds to the illustration on the right. Arrows direct attention to the anatomic feature of interest: (A) bilateral aplastic P1s, (B) duplicated PCoA take off, (C) bilateral duplicated/accessory MCAs, (D) fenestrated M1, and (E) bilateral hypoplastic proximal MCAs.

(Komiyama et al., 1997; Liu et al., 2015). Thus, it is plausible that in mice, duplicated MCAs could potentially lead to smaller infarct volumes following MCAO.

\section{Study Limitations}

This study has important limitations. A total of 144 mice were included, with as few as three mice per strain, limiting the 
generalizability of these results. We can thus draw conclusions regarding variations in vessel diameters across strain but not within strains. The precision of the measurements is limited by potential subtle variations in India ink perfusion and by image resolution. To maximize reproducibility, we have performed the India ink perfusion using heated ink and perfused under pressures of $60-80 \mathrm{~mm} \mathrm{Hg}$. An adequate view of the P1 segments was often limited as these segments were sometimes hidden from view near the junction of the pons and midbrain. Thus, we are unable to rule out the presence of P1 segments. These data do not demonstrate any functional differences; rather they document variations in the diameters of these vessels.

In summary, this study investigated anatomic differences in the caliber of cerebral arteries between 32 inbred strains of mice, taking advantage of a large number of strains to evaluate the impact of strain on artery size, which has not been done to this extent. These data suggest mouse cerebral artery diameter is determined by genetics rather than the mean size of the adult mouse, as evidenced by variations due to strain and not weight. Thus, data from one strain should be interpreted with caution when compared to mice from different strains. To increase the robustness of these data, a larger number of mice per strain

\section{REFERENCES}

Barbeito-Andrés, J., Bernal, V., and Gonzalez, P. N. (2016). Morphological asymmetries of mouse brain assessed by geometric morphometric analysis of mri data. Magn. Reson. Imaging 34, 980-989. doi: 10.1016/j.mri.2016. 04.006

Barone, F. C., Knudsen, D. J., Nelson, A. H., Feuerstein, G. Z., and Willette, R. N. (1993). Mouse strain differences in susceptibility to cerebral ischemia are related to cerebral vascular anatomy. J. Cereb. Blood Flow Metab. 13, 683-692. doi: $10.1038 /$ jcbfm.1993.87

Beckmann, N. (2000). High resolution magnetic resonance angiography non-invasively reveals mouse strain differences in the cerebrovascular anatomy in vivo. Magn. Reson. Med. 44, 252-258. doi: 10.1002/15222594(200008)44:2<252::AID-MRM12>3.0.CO;2-G

Benjamini, Y., and Yekutieli, D. (2001). The control of the false discovery rate in multiple testing under dependency. Ann. Stat. 29, 1165-1188. doi: 10.1214/aos/1013699998

Bühler, D., Schüller, K., and Plesnila, N. (2014). Protocol for the induction of subarachnoid hemorrhage in mice by perforation of the circle of willis with an endovascular filament. Transl. Stroke Res. 5, 653-659. doi: 10.1007/s12975-014-0366-6

Casals, J. B., Pieri, N. C., Feitosa, M. L., Ercolin, A. C., Roballo, K. C., Barreto, R. S., et al. (2011). The use of animal models for stroke research: a review. Comp. Med. 61, 305-313.

Du, R., Zhou, J., Lorenzano, S., Liu, W., Charoenvimolphan, N., Qian, B., et al. (2015). Integrative mouse and human studies implicate angptl and zbtb7c as susceptibility genes to ischemic injury. Stroke 46, 3514-3522. doi: 10.1161/STROKEAHA.115.010767

Fox, G., Gallacher, D., Shevde, S., Loftus, J., and Swayne, G. (1993). Anatomic variation of the middle cerebral artery in the sprague-dawley rat. Stroke 24, 2087-2092. discussion: 2092-2083. doi: 10.1161/01.STR.24.12.2087

Heine, O., and Galaburda, A. M. (1986). Olfactory asymmetry in the rat brain. Exp. Neurol. 91, 392-398. doi: 10.1016/0014-4886(86)90078-6

Hata, R., Mies, G., Wiessner, C., Fritze, K., Hesselbarth, D., Brinker, G., et al. (1998). A reproducible model of middle cerebral artery occlusion in mice: hemodynamic, biochemical, and magnetic resonance imaging. J. Cereb. Blood Flow Metab. 18, 367-375. doi: 10.1097/00004647-19980400000004 will be required in future studies. Additional studies will also be necessary to determine the underlying genetic mechanisms driving cerebral artery diameters.

\section{AUTHOR CONTRIBUTIONS}

BQ: Acquired data, critically revised the manuscript; RR: Acquired and analyzed data, drafted the manuscript; TC: Analyzed the data, critically revised the manuscript; RD: Conceived of and designed the study, critically revised the manuscript.

\section{FUNDING}

This work was supported by the National Institutes of Health grant K08NS067172 (RD).

\section{SUPPLEMENTARY MATERIAL}

The Supplementary Material for this article can be found online at: https://www.frontiersin.org/articles/10.3389/fnana. 2018.00010/full\#supplementary-material

Chang, H. Y., and Kim, M. S. (2011). Middle cerebral artery duplication : classification and clinical implications. J. Korean Neurosurg. Soc. 49, 102-106. doi: 10.3340/jkns.2011.49.2.102

Kitagawa, K., Matsumoto, M., Yang, G., Mabuchi, T., Yagita, Y., Hori, M., et al. (1998). Cerebral ischemia after bilateral carotid artery occlusion and intraluminal suture occlusion in mice: evaluation of the patency of the posterior communicating artery. J. Cereb. Blood Flow Metab. 18, 570-579. doi: 10.1097/00004647-199805000-00012

Komiyama, M., Nakajima, H., Nishikawa, M., and Yasui, T. (1998). Middle cerebral artery variations: duplicated and accessory arteries. AJNR. Am. J. Neuroradiol. $19,45-49$.

Komiyama, M., Nishikawa, M., and Yasui, T. (1997). The accessory middle cerebral artery as a collateral blood supply. AJNR. Am. J. Neuroradiol. 18, 587-590.

Liu, Z. S., Zhou, L. J., Sun, Y., Kuang, X. W., Wang, W., and Li, C. (2015). Sufficient collateral blood supply from accessory middle cerebral artery in a patient with acute ischemic stroke. Interv. Neuroradiol. 21, 215-217. doi: $10.1177 / 1591019915583230$

Maeda, K., Hata, R., and Hossmann, K. A. (1998). Differences in the cerebrovascular anatomy of c57black/6 and sv129 mice. Neuroreport 9, 1317-1319. doi: 10.1097/00001756-199805110-00012

Majid, A., He, Y. Y., Gidday, J. M., Kaplan, S. S., Gonzales, E. R., Park, T. S., et al. (2000). Differences in vulnerability to permanent focal cerebral ischemia among 3 common mouse strains. Stroke 31, 2707-2714. doi: 10.1161/01.STR.31.11.2707

McGirt, M. J., Lynch, J. R., Parra, A., Sheng, H., Pearlstein, R. D., Laskowitz, D. T., et al. (2002). Simvastatin increases endothelial nitric oxide synthase and ameliorates cerebral vasospasm resulting from subarachnoid hemorrhage. Stroke 33, 2950-2956. doi: 10.1161/01.STR.0000038986.68044.39

Morimoto, M., Miyamoto, S., Mizoguchi, A., Kume, N., Kita, T., and Hashimoto, N. (2002). Mouse model of cerebral aneurysm: experimental induction by renal hypertension and local hemodynamic changes. Stroke 33, 1911-1915. doi: 10.1161/01.STR.0000021000.19637.3D

Neuwirth, E. (2014). Rcolorbrewer: Colorbrewer Palettes. R package version 1. 1-2.

Nishio, K., Ihara, M., Yamasaki, N., Kalaria, R. N., Maki, T., Fujita, Y., et al. (2010). A mouse model characterizing features of vascular dementia with hippocampal atrophy. Stroke 41, 1278-1284. doi: 10.1161/STROKEAHA.110. 581686 
Orset, C., Macrez, R., Young, A. R., Panthou, D., Angles-Cano, E., Maubert, E., et al. (2007). Mouse model of in situ thromboembolic stroke and reperfusion. Stroke 38, 2771-2778. doi: 10.1161/STROKEAHA.107.487520

Percie du Sert, N., Alfieri, A., Allan, S. M., Carswell, H. V., Deuchar, G. A., Farr, T. D., et al. (2017). The improve guidelines (ischaemia models: procedural refinements of in vivo experiments). J. Cereb. Blood Flow Metab. 37, 3488-3517. doi: 10.1177/0271678X17709185

Purcell, S., Neale, B., Todd-Brown, K., Thomas, L., Ferreira, M. A., Bender, D., et al. (2007). Plink: a tool set for whole-genome association and population-based linkage analyses. Am. J. Hum. Genet. 81, 559-575. doi: 10.1086/519795

R Core Team (2017). R: A Language and Environment for Statistical Computing, Version 3, 4. Vienna.

Robinson, D. (2017). Broom: Convert Statistical Analysis Objects into Tidy Data Frames. R package version 0.4.2.

Roth, G. A., Huffman, M. D., Moran, A. E., Feigin, V., Mensah, G. A., Naghavi, M., et al. (2015). Global and regional patterns in cardiovascular mortality from 1990 to 2013. Circulation 132, 1667-1678. doi: 10.1161/CIRCULATIONAHA.114.008720

Rynkowski, M. A., Kim, G. H., Komotar, R. J., Otten, M. L., Ducruet, A. F., Zacharia, B. E., et al. (2008). A mouse model of intracerebral hemorrhage using autologous blood infusion. Nat. Protoc. 3, 122-128. doi: 10.1038/nprot.2007.513

Schneider, C. A., Rasband, W. S., and Eliceiri, K. W. (2012). Nih image to imagej: 25 years of image analysis. Nat. Methods 9, 671-675. doi: 10.1038/nmeth.2089

Schomer, D. F., Marks, M. P., Steinberg, G. K., Johnstone, I. M., Boothroyd, D. B., Ross, M. R., et al. (1994). The anatomy of the posterior communicating artery as a risk factor for ischemic cerebral infarction. N. Engl. J. Med. 330, 1565-1570. doi: 10.1056/NEJM199406023302204

Sheldon, R. A., Sedik, C., and Ferriero, D. M. (1998). Strain-related brain injury in neonatal mice subjected to hypoxia-ischemia. Brain Res. 810, 114-122. doi: 10.1016/S0006-8993(98)00892-0

Ward, R., Collins, R. L., Tanguay, G., and Miceli, D. (1990). A quantitative study of cerebrovascular variation in inbred mice. J. Anat. 173, 87-95.

Warnes, G. R., Bolker, B., Bonebakker, L., Gentleman, R., Liaw, W. H. A., Lumley, T., et al. (2016). Gplots: Various R Programming Tools For Plotting Data. $\mathrm{R}$ package version 3.0.1.
Wellons, J. C. III., Sheng, H., Laskowitz, D. T., Mackensen, G. B., Pearlstein, R. D., and Warner, D. S. (2000). A comparison of strain-related susceptibility in two murine recovery models of global cerebral ischemia. Brain Res. 868, 14-21. doi: 10.1016/S0006-8993(00)02216-2

Wickham, H. (2009). Ggplot2: Elegant Graphics for Data Analysis. New York, NY: Springer-Verlag.

Wickham, H. (2011). The split-apply-combine strategy for data analysis. J. Stat. Softw. 40, 1-29. doi: 10.18637/jss.v040.i01

Wickham, H. (2017a). Stringr: Simple, Consistent Wrappers for Common String Operations. R package version 1.2.0.

Wickham, H. (2017b). Tidyr: Easily Tidy Data with 'Spread()' and 'Gather()' Functions. R package version 0.6.3.

Wickham, H., Francois, R., Henry, L., and Müller, K. (2017). Dplyr: A Grammar of Data Manipulation. R package version 0.7.1.

Xue, S., Gong, H., Jiang, T., Luo, W., Meng, Y., Liu, Q., et al. (2014). Indianink perfusion based method for reconstructing continuous vascular networks in whole mouse brain. PLoS ONE 9:e88067. doi: 10.1371/journal.pone.00 88067

Zeal, A. A., and Rhoton, A. L. Jr. (1978). Microsurgical anatomy of the posterior cerebral artery. J. Neurosurg. 48, 534-559. doi: 10.3171/jns.1978.48. 4.0534

Zhang, H., Prabhakar, P., Sealock, R., and Faber, J. E. (2010). Wide genetic variation in the native pial collateral circulation is a major determinant of variation in severity of stroke. J. Cereb. Blood Flow Metab. 30, 923-934. doi: $10.1038 /$ jcbfm.2010.10

Conflict of Interest Statement: The authors declare that the research was conducted in the absence of any commercial or financial relationships that could be construed as a potential conflict of interest.

Copyright (C) 2018 Qian, Rudy, Cai and Du. This is an open-access article distributed under the terms of the Creative Commons Attribution License (CC BY). The use, distribution or reproduction in other forums is permitted, provided the original author(s) and the copyright owner are credited and that the original publication in this journal is cited, in accordance with accepted academic practice. No use, distribution or reproduction is permitted which does not comply with these terms. 